\title{
Risk Factors for Parental Burnout among Finnish Parents: The Role of Socially Prescribed Perfectionism
}

\author{
Matilda Sorkkila $\mathbb{1}^{1} \cdot$ Kaisa Aunola ${ }^{1}$
}

Published online: 10 October 2019

(c) The Author(s) 2019

\begin{abstract}
Objectives Although parental burnout can have detrimental consequences to families, the investigation of the syndrome is still in its infancy. The present study investigated what are the key family background variables that contribute to parental burnout among Finnish parents. Moreover, we investigated how self-oriented and socially prescribed perfectionism uniquely and interactively relate to parental burnout over and above the impacts of background variables.

Methods Questionnaire-based data was collected from 1725 parents (91\% mothers) and analyzed using structural equation modeling (SEM).

Results The results showed that when several family- and child-related background variables were taken into account, parent's age, unemployment, perceived poor financial situation of the family, and having a child with special needs, showed unique associations with burning out as a parent. Even more crucial risk factor for burning out was, however, socially prescribed perfectionism: the higher the level of socially prescribed perfectionism the parents reported, the higher the level of their parental burnout. The relationship between socially prescribed perfectionism and parental burnout was further strengthened when parents reported also a high level of self-oriented perfectionism. Finally, the relationship between gender and parental burnout was mediated via perfectionism: mothers reported more socially prescribed and self-oriented perfectionism than fathers and, consequently, were also more burned out as parents.

Conclusions The results suggest that in Finland specific attention should be given to families with poor financial resources and unemployment. Moreover, high social expectations experienced by the mothers could be balanced, for example, by teaching them skills of self-acceptance and compassion.
\end{abstract}

Keywords Family background variables $\cdot$ Parental exhaustion $\cdot$ Self-expectations $\cdot$ Social expectations $\cdot$ Structural equation modeling

Although it is well known that parenting can be stressful and tiring (Crnic and Low 2002; Deater-Deckard 2008), only recently has parental burnout been acknowledged as its independent psychological phenomenon (Roskam et al. 2018; Roskam et al. 2017). Parental burnout—defined as exhaustion in one's role as a parent, feelings of being fed up as a parent, and emotional distancing from one's children (Roskam et al. 2018) — has been associated with various serious outcomes, such as addictive behaviors, health disorders, couple's conflicts, escape, and suicidal ideation

Matilda Sorkkila

matilda.sorkkila@jyu.fi

1 Department of Psychology, University of Jyvaskyla, P. O. Box 35, FI-40014 Jyvaskyla, Finland
(Mikolajczak et al. 2018a). Furthermore, it has been shown to increase the risk of violence and abuse towards one's children (Mikolajczak et al. 2018a). To address burnout prevention and treatment, it is crucial to identify the factors behind parental burnout. These factors may include sociodemographic background factors (e.g., financial situation of the family, the age of parent, and the number of children) and children's characteristics (e.g., age of children, developmental delays, or permanent illnesses) (Lindhal-Norberg 2007; Lindström et al. 2011). Moreover, personality characteristics may also play a role (Kawamoto et al. 2018; Le Vigouroux and Scola 2018; Le Vigouroux et al. 2018). One personality characteristic which may be particularly crucial in the development of burnout is multidimensional perfectionism (for a review, see Hill and Curran 2016) consisting of self-oriented perfectionism (i.e., high self-standards accompanied by harsh self-criticism) and socially 
prescribed perfectionism (i.e., perceived high standards from others; Hewitt and Flett 1991). Although parents have been shown to be both harsh on themselves and experience pressure in their parenting from other people (Douglas and Michaels 2004; Hagger 2011), the relationship between parental burnout and multidimensional perfectionism has so far not been investigated. Furthermore, studies including both family background and children's characteristics, as well as parental personality factors as predictors of parental burnout are rare (Kawamoto et al. 2018; Le Vigouroux and Scola 2018; Mikolajczak et al. 2018b).

Burnout has been largely studied in a work context, and it has been defined as a condition resulting from chronic stress and characterized by exhaustion (i.e., chronic fatigue), cynicism (i.e., callous and distant attitude towards work), and a lack of professional efficiency (i.e., feelings of incompetence and lack of usefulness) (Maslach and Jackson 1986; Maslach et al. 2001). Since then, it has been argued that burnout can occur in any environment characterized by enduring chronic stress (Bianchi et al. 2014). For example, Roskam et al. (2017) demonstrated that burnout could occur in the context of parenting, identifying parental burnout as a separate and unique syndrome that is both theoretically and practically different from work burnout (Roskam et al. 2017, 2018). Based on their groundbreaking research, Roskam et al. (2018) conceptualized four dimensions of parental burnout: (1) exhaustion related to one's role as a parent (i.e., feelings that parenting requires too much involvement; emotionally draining role as a parent), (2) contrast with previous parental self (i.e., feelings that one is not as good of a parent as in the past; shame regarding one's parenting), (3) feelings of being fed up with one's parental role (i.e., not enjoying spending time with one's children anymore; not happy with the parenting role), and (4) emotional distancing from one's children (i.e., doing the bare minimum for the children and nothing more; limiting interactions to instrumental aspects of parenting at the cost of emotional aspects).

It has been suggested that parental burnout develops as a consequence of chronic exposure to parental stress, where the experienced demands (i.e., risk factors) constantly exceed the available resources (i.e., protective factors; Mikolajczak and Roskam 2018). This theory is based on the demands-resources model used in organizational psychology (Bakker and Demerouti 2007; Demerouti et al. 2001), according to which job burnout develops when demands are high and resources are low, which results in energy depletion that undermines an employee's motivation (Demerouti et al. 2001). In a parenting context (Mikolajczak and Roskam 2018), the demands are stress-increasing factors, such as countless parental chores and duties or a lack of external support (i.e., support from nurseries, family support). The resources are, in turn, stress-decreasing factors, such as time for leisure activities or relaxation or external support.

Several family background factors can be considered to act as risk factors for parental burnout (see Mikolajczak et al. 2018b), such as having many children (large families may increase parental demands) (Lundberg et al. 1994), having young children (younger children need more looking after than older children do), having a small age gap between children (requiring more parental investment), being female (mothers tend to be more involved in childcare than fathers) (Lindhal-Norberg 2007), being a single parent (single parents may not be able to share chores or responsibilities with anyone), having a blended family (parents may have authority issues or other difficulties with stepchildren) (Baxter et al. 2004), low financial resources of the family (parents with lower income suffer from additional money-related stress, and they may not be able to afford additional services such as babysitting), parental unemployment (being unemployed may cause additional stress and affect self-esteem), or having a child with special needs (such children require extra attention and care) (Blanchard et al. 2006; Mikolajczak et al. 2018b). However, a recent study of Belgian parents revealed that sociodemographic factors explained less variance in parental burnout than particularities of the child, parents' personality traits, parenting factors, and family functioning (Mikolajczak et al. 2018b). In the study, the only sociodemographic factor that was a significant contributor to parental burnout was having a child under the age of five. Conversely, another recent study carried out among French parents showed that the age of the parent, the total number of children, and the gap in children's age were risk factors for parental burnout (i.e., being a young parent, having a large family, having children with large age gaps), even when investigated along with the personality traits of the parents and children (Le Vigouroux and Scola 2018). Similarly, the results of a study of Japanese parents demonstrated that younger parents experienced more burnout than older parents, and having more children increased the risk of burnout (Kawamoto et al. 2018), although in line with the other studies (Mikolajczak et al. 2018b), sociodemographic factors explained relatively little of the variance in parental burnout.

Since previous research has shown that sociodemographic factors may explain only a portion of the variance in parental burnout, the question arises as to whether parental personality-related characteristics could play a larger role (Lindström et al. 2011; Mikolajczak et al. 2018b). One personality factor that has been shown to be a significant predictor of burnout in occupational, athletic, and educational settings is perfectionism (for a review, see Hill and Curran 2016). Perfectionism has been defined as an individual's tendency to set extremely high self-standards and strive for flawless performance accompanied by critical 
self-evaluations (Flett and Hewitt 2002; Frost et al. 1990). Hewitt and Flett (1991) divided perfectionism into selforiented and socially prescribed perfectionism (see also, Hill et al. 2008). Self-oriented perfectionism is characterized by extremely high self-standards accompanied by harsh self-criticism, whereas socially prescribed perfectionism is characterized by beliefs that others impose high or even unrealistic standards, and that one can be accepted by others only by fulfilling these standards (Hewitt and Flett 1991). Previous research in sport context has shown that while socially prescribed perfectionism is generally thought of as debilitating because it is associated with self-criticism, selfblame, anxiety, and overgeneralization of failure (Flett et al. 1991), self-oriented perfectionism predisposes individuals to negative outcomes only when interacting with some other variables (see Hill et al. 2008). For example, although there are studies showing that self-oriented perfectionism is associated with burnout (see Hill et al. 2008), there is also evidence that in some circumstances self-oriented perfectionism may lead to positive outcomes, such as intrinsic motivation, striving for achievement (Mills and Blankstein 2000), and resourcefulness (Flett et al. 1991). It is also possible that the two forms of perfectionism interact (Flett and Hewitt 2002). For example, research carried out in clinical setting has demonstrated that it is the combination of two forms of perfectionism rather than either one alone that is most detrimental in terms of disordered eating (Esposito et al. 2016) Thus far, however, only few studies have examined the interactive effects of the self-oriented and socially prescribed perfectionism (e.g., Appleton et al. 2009; Esposito et al. 2016), and to the best of our knowledge, none of these have been carried out among parents.

In addition to the multidimensional definition of perfectionism by Hewitt and Flett (1991), other conceptualizations of perfectionism have been utilized as well. For example, Frost et al. (1990) divided perfectionism into perfectionistic strivings and concerns: Whereas perfectionistic strivings consist of high personal standards and striving for perfection, perfectionistic concerns consist of negative reactions to imperfection, fear about failing to meet other's expectations, and concern over mistakes. Nevertheless, this conceptualization has been criticized of being difficult to interpret, as it allows viewing perfectionism also in the absence of concerns over mistakes, which is a fundamental aspect of the construct (see Hill et al. 2008). This critique is further supported by the findings demonstrating that perfectionistic strivings and concerns correlate strongly with each other (Hill et al. 2008; Kawamoto et al. 2018).

Thus far, only one study has investigated the role of perfectionism in burnout in a parental context (Kawamoto et al. 2018), and this study used the definition of Frost et al. (1990) to capture perfectionism. The results of this study showed that perfectionistic concerns (i.e., concerns regarding mistakes and negative evaluations; Frost et al. 1990), in particular, was a strong predictor of parental burnout among Japanese parents. In fact, perfectionistic concerns explained significantly more variance in parental burnout than in occupational burnout. The results could be explained by the perfectionistic parents' tendency to overgeneralize negative events, ruminate about past failures, and engage in all-or-nothing thinking (Flett and Hewitt 2002; Kawamoto et al. 2018). Although the concept of perfectionistic concerns (Frost et al. 1990) is not directly comparable to socially prescribed perfectionism (Hewitt and Flett 1991), these two concepts share the component of fear over others' negative evaluations and, thus, may be similarly related to parental burnout. Moreover, if one feels like they are accepted by others only if they meet their high standards (i.e., socially prescribed perfectionism), they may be particularly vulnerable to concerns related to mistakes and negative reactions to imperfectionism (i.e., perfectionistic concerns). Based on previous literature and the demands-resources model (Bakker and Demerouti 2007; Demerouti et al. 2001), the high standards an individual sets for her- or himself (selforiented perfectionism) as well as the perceived high demands of others and the related worries about negative evaluations can be considered to be stress-increasing factors in a parenting context. For example, it has been suggested that perfectionism may be closely linked to parental meticulousness, as the parents may exhaust themselves by trying to do "too well" (Lindström et al. 2011). Because socially prescribed perfectionism is closely related to neuroticism and negative affect (e.g., Flett and Hewitt 2002; Molnar et al. 2006) as well as to anxiety and depression (Flett and Hewitt 2002), all of which are also linked to parental burnout (see Mikolajczak et al. 2018b), this form of perfectionism, in particular, could be assumed to play a role in parental burnout.

Overall, only few studies have examined different antecedents of parental burnout (e.g., sociodemographic and personality factors as well as child characteristics) in the same study (Le Vigouroux and Scola 2018; Lindström et al. 2011; Mikolajczak et al. 2018b). Thus, little is known about the relative role of these different variables in parental burnout. Moreover, previous studies have been carried out only with French (Le Vigouroux and Scola 2018; Le Vigouroux et al. 2018), Belgian (e.g., Mikolajczak et al. 2018a; 2018b, Roskam et al. 2018), Swedish (Lindström et al. 2011, 2010), and Japanese (Kawamoto et al. 2018) parents. Because the role of each sociodemographic background variable on parental burnout may differ between countries and cultures (see, for example Kawamoto et al. 2018; Le Vigouroux and Scola 2018; Mikolajczak et al. 2018b), studies in different cultural settings are needed to get a broader understanding of 
the phenomenon. For example, in Finland, it is typical for people to live far away from grandparents and other social support networks due to studies or employment (Salmi and Lammi-Taskula 2014). Therefore, having many children or having a young child in the house may contribute more to parental burnout in Finland than in other cultures in which natural social support is normally available. Moreover, in Finland, gender-equality is encouraged in both parenting and working life (LammiTaskula 2017). A recent cross-cultural study (Hagqvist et al. 2017) showed that parents in countries with high level of gender equality in working life (e.g., Finland) had more difficulties integrating work and family life and reported less well-being than parents in countries with more traditional gender roles (e.g., Czech Republic). This was shown to be particularly detrimental for mothers, who are often expected work equally to men and earn money, but who still carry the main responsibility of children and home (Hagqvist et al. 2017). Consequently, there may be structural and value-based differences in the social systems across countries, which may impact burning out as parent differently.

The aim of the present study was, first, to identify the key family background variables that explain parental burnout among Finnish parents. We assumed that family background variables would explain only a small amount of the variance in parental burnout (Hypothesis 1). Second, we aimed to examine how different forms of perfectionism, that is, self-oriented and socially prescribed perfectionism, are related to parental burnout over and above the impacts of background variables. We hypothesized that particularly socially prescribed perfectionism would be positively associated with parental burnout (Hypothesis 2), and that a high level of self-oriented perfectionism would strengthen the relationship between socially prescribed perfectionism and parental burnout (Hypothesis 3).

\section{Method}

\section{Participants}

The participants were 1725 Finnish parents (91\% mother) who had at least one child living with them (either permanently or part-timely) in the same household. The parents' average age was $36(S D=6.50)$. The youngest child in the family was, on average, 4 years of old $(S D=4.15)$ and the oldest child eight $(S D=5.32)$. The number of children in the participating families ranged from one to $17(M=2.25$, $S D=1.34)$, the number of biological children ranging from 0 to $17(M=2.20, S D=1.20)$.

From the participants, $99.1 \%$ were native Finnish. The parents completed either online $(86 \%)$ or pen-and-paper questionnaires at Child Health Centers in three Finnish cities $(13 \%)$ or in congregational family playgroups $(1 \%)$. The Child Health Centers were selected based on geographical representativeness (the cities represent Southern, Middle, and Northern Finland), and they were considered appropriate locations for reaching a heterogeneous sample of parents (e.g., including different kinds of family types from different socioeconomic classes) since all Finnish parents are required to take their 0-6-year-old-children for annual check-ups in these centers. Due to the small number of participating fathers, two family playgroups were also included, which had specific playgroups for fathers and children. Web questionnaires were selected due to their ability to reach a large number of parents from different sides of Finland.

A total of $78.7 \%$ parents lived in a nuclear family, $9.6 \%$ lived in a single parent household, and $8.7 \%$ lived in a blended family. A total of $74.2 \%$ of the participants had a university or college degree, $8.4 \%$ had a technical college degree, $14.7 \%$ had a vocational school degree, and $2.7 \%$ had no vocational degree. Examination of relevant background variables (e.g., level of education, number of children) of the present study revealed that parents with higher education (a university or college degree) were overrepresented $(74.2 \%)$ in the sample compared to general Finnish population (44.4\%; Official Statistics of Finland 2018a, b). Compared to general Finnish population (Official Statistics of Finland 2018a, b), the number of children in the present sample was also somewhat higher.

\section{Procedure}

Ethical permission for the study was obtained from the ethical committee of the University of Jyväskylä. Prior to their participation, all the participants provided informed consent to confirm their voluntary participation in the study. The web questionnaire was advertised through different social media channels, and parents could participate by clicking the link. At the Child Health Centers, the nurses introduced the study to the parents at the end of their annual check-up, and after the check-up, the parents were asked to complete the questionnaire in the waiting room and then drop it anonymously into a post box marked with the project's name. Alternatively, the parents were given the option to take the questionnaire home and send it back to the researchers anonymously in a pre-paid envelope. In the family playgroups, the instructors introduced the study to the parents. When the playgroups ended, the parents were asked to complete the questionnaire and return it to the researchers anonymously in a pre-paid envelope. All answers were inserted either electronically (web-based questionnaires) or manually (paper questionnaires) into the IBM SPSS Statistical software program (Version 24). 


\section{Measures}

\section{Parental burnout}

Parental burnout was measured using the Parental Burnout Assessment (PBA) (Roskam et al. 2018) which was translated to Finnish and validated in Finland by the authors (Aunola et al. 2019). The scale consists of 23 items: nine measure exhaustion in the parental role (e.g., $I$ feel completely run down by my role as a parent), six measure contrast in the parental self (e.g., I don't think I'm the good father/mother that I used to be to my children), five measure feelings of being fed up as a parent (e.g., I can't stand my role as father/mother anymore), and three measure emotional distancing from one's children (e.g., I do what I'm supposed to do for my children but nothing more). All items were rated on a 7-point Likert scale indicating how often the parent feels a certain way $(0=$ never; $6=$ every day). Cronbach's alpha reliabilities for the subscales were 0.95, 0.92, 0.86, and 0.75, respectively.

\section{Self-oriented and socially prescribed perfectionism}

Multidimensional perfectionism was measured using two different subscales: self-oriented perfectionism (4 items; e.g., It is important for me to be perfect in everything I attempt) and socially prescribed perfectionism (3 items; e.g., People expect too much from me), taken from the Big Three Perfectionism Scale (Smith et al. 2016), in which the items of Hewitt and Flett (1991) are modified into a general context. The scale was translated to Finnish by the authors. Parents were asked to answer each item using a 5-point Likert scale $(1=$ completely disagree; $5=$ completely agree). Cronbach's alpha reliabilities for self-oriented perfectionism and socially prescribed perfectionism were 0.70 and 0.80 , respectively.

\section{Family- and child-related background variables}

In the present study, the following family- and child-related background variables were used:

Parent's employment status was assessed with the question "Do you have a paid professional activity?" The parents answered by selecting either "yes" (value 1) or "no" (value 2). For statistical analysis, the variable was reversed so that a higher value described employment and a lower value unemployment. A total of $75.6 \%$ of the parents were employed and $24.4 \%$ were unemployed.

Parental level of education was assessed with the question "Which is the highest level of education you have?" The parents answered by selecting one option from the following alternatives: $1=$ no vocational education, $2=$ vocational school degree, $3=$ technical college degree, and $4=$ university or college degree. For statistical analyses, four dichotomous variables were created based on the variable to describe whether the parent had a particular level of education $($ yes $=1)$ or not $($ no $=0)$.

Family income was assessed by asking the parents how they perceived the financial situation of their family. The parents selected one of the following options: $1=$ poor (the option reported by $5.2 \%$ of the participants), $2=$ poorer than average (19.2\%), $3=$ average $(52.4 \%)$, and $4=$ higher than average $(23.2 \%)$. For statistical analyses, four dichotomous variables were created based on the variable to describe whether the parent reported a particular level of financial situation (yes $=1)$ or not $($ no $=0)$.

Information concerning developmental delays or special needs of any child in the home was gathered with the question "Do any of the children in your family currently have special needs (e.g., colic, developmental delays, or neurological or conduct disorders)?" The parents answered the question by selecting either the option "no" (value 1) or "yes" (value 2). A total of $23.8 \%$ of the participants reported special deeds among their children.

Parents' gender was assessed by asking: Are you (1) a father or a similar type of guardian (e.g., a stepfather); (2) a mother or a similar type of guardian (e.g., a step mother). Parents' age was assessed with an open question. In addition, open questions were used to find the ages of the youngest and oldest children living at home. Based on this information, a new variable was created: at least one young child at home $($ age $<6)$ vs. no young children (age of the youngest child $>5$ ) at home. This categorization was selected to be consistent with the previous literature on the predictors of parental burnout (Mikolajczak et al. 2018b).

A "Single parenthood" variable $(1=$ single parent raising children alone, $0=$ other type of family) was formed based on the question concerning family type ("What type is your family?"), for which the parents selected the suitable option from six different family types $(1=$ nuclear family; $2=\mathrm{a}$ single parent raising children alone; $3=$ step-family; $4=$ rainbow family; $5=$ family of several generations; $6=$ other type of family). Based on the family type variable, a new variable was also created to describe blended families ( $1=$ step-family, $0=$ other type of family).

Finally, time spent with children during the day was assessed with the open question "On average, how many hours a day do you spend with your child(ren) (without taking the night into account)?"

\section{Data Analyses}

In the present study, the analyses were carried out using structural equation modeling with Mplus statistical software (version 8) (Muthén and Muthén 2017). The 
parameters of the models were estimated using the MLR estimator, which corrects the standard errors in the case of non-normality. By applying a missing-data method, all available data was used to estimate the model without inputting data. The analyses were conducted in the following five steps: First, as a preliminary analysis, bivariate correlations between all study variables were examined. Second, separate measurement models for latent parental burnout and multidimensional perfectionism were tested. Third, a structural equation model (SEM) examining the unique role of different family background variables in the latent parental burnout was conducted. Fourth, latent factors indicating parents' self-oriented and socially prescribed perfectionism were included into the previous model as predictors of parental burnout. Finally, the interaction term Socially prescribed perfectionism $\mathrm{X}$ Self-oriented perfectionism was tested by including the effect of this interaction term on latent parental burnout. The means, standard deviations, and correlations between the study variables are shown in Table 1.

\section{Results}

Inspection of correlations (Table 1) demonstrated that the two forms of perfectionism correlated positively and moderately with each other $(r=0.37)$, suggesting that selforiented and socially prescribed perfectionisms are positively related but still different constructions. From the hypothesized predictor variables of parental burnout, the highest correlations were between perfectionism variables and parental burnout, higher level of perfectionism being associated with higher levels of parental burnout in both cases of perfectionism. From other predictor variables, special needs among children, perceived family financial situation, and employment status, respectively, were the variables most strongly associated with parental burnout. Gender, single parenthood, time spent with children and, finally, parent's age, were also statistically significantly related to burnout, although to lesser extent.

The modeling of the results was started by testing separate measurement models for latent parental burnout (4 measured

Table 1 Means $^{3}(M)$, Standard Deviations (SD), and Correlations (Pearson Moment Correlations for Continuous Variables and Spearman Correlations for Categorical Variables ${ }^{1}$ ) between Study Variables

\begin{tabular}{|c|c|c|c|c|c|c|c|c|c|c|c|c|c|c|}
\hline & 1. & 2. & 3. & 4. & 5. & 6. & 7. & 8. & 9. & 10. & 11. & 12. & 13. & 14. \\
\hline 1. Gender ${ }^{1}$ & 1.00 & & & & & & & & & & & & & \\
\hline 2. Age & -0.04 & 1.00 & & & & & & & & & & & & \\
\hline 3. Level of education ${ }^{1}$ & $0.05^{\mathrm{c}}$ & $0.12^{\mathrm{a}}$ & 1.00 & & & & & & & & & & & \\
\hline $\begin{array}{l}\text { 4. Family financial } \\
\text { situation }^{1}\end{array}$ & -0.00 & $0.16^{\mathrm{a}}$ & $0.26^{\mathrm{a}}$ & 1.00 & & & & & & & & & & \\
\hline 5. Employment ${ }^{1}$ & $-0.12^{\mathrm{a}}$ & $0.26^{\mathrm{a}}$ & $0.15^{\mathrm{a}}$ & $0.26^{\mathrm{a}}$ & 1.00 & & & & & & & & & \\
\hline 6. Single parenthood ${ }^{1}$ & $0.08^{\mathrm{b}}$ & $0.13^{\mathrm{a}}$ & $-0.07^{b}$ & $-0.23^{\mathrm{a}}$ & -0.00 & 1.00 & & & & & & & & \\
\hline 7. Blended family ${ }^{1}$ & -0.05 & $0.08^{\mathrm{b}}$ & $-0.12^{\mathrm{a}}$ & $-0.09^{\mathrm{a}}$ & -0.00 & - & 1.00 & & & & & & & \\
\hline 8. Number of children & 0.00 & $0.17^{\mathrm{a}}$ & $-0.08^{\mathrm{b}}$ & 0.03 & -0.01 & $-0.13^{\mathrm{a}}$ & $0.28^{\mathrm{a}}$ & 1.00 & & & & & & \\
\hline $\begin{array}{l}\text { 9. Young child }(<6) \text { in the } \\
\text { family } 1\end{array}$ & $-0.06^{\mathrm{c}}$ & $-0.52^{\mathrm{a}}$ & 0.00 & -0.02 & $-0.20^{\mathrm{a}}$ & $-0.20^{\mathrm{a}}$ & $-0.11^{\mathrm{a}}$ & $-0.06^{\mathrm{c}}$ & 1.00 & & & & & \\
\hline $\begin{array}{l}\text { 10. Special needs among } \\
\text { children }^{1}\end{array}$ & $0.08^{b}$ & $0.18^{\mathrm{a}}$ & $-0.10^{\mathrm{a}}$ & $-0.10^{\mathrm{a}}$ & -0.01 & $0.11^{\mathrm{a}}$ & $0.15^{\mathrm{a}}$ & $0.17^{\mathrm{a}}$ & $-0.19^{\mathrm{a}}$ & 1.00 & & & & \\
\hline $\begin{array}{l}\text { 11. Time spend with } \\
\text { children }\end{array}$ & $0.17^{\mathrm{a}}$ & $-0.33^{\mathrm{a}}$ & $-0.09^{\mathrm{a}}$ & $-0.12^{\mathrm{a}}$ & $-0.49^{\mathrm{a}}$ & $-0.06^{\mathrm{c}}$ & -0.04 & $0.12^{\mathrm{a}}$ & $0.40^{\mathrm{a}}$ & -0.04 & 1.00 & & & \\
\hline $\begin{array}{l}\text { 12. Self-oriented } \\
\text { perfectionism }\end{array}$ & $0.07^{\mathrm{b}}$ & $-0.13^{\mathrm{a}}$ & -0.05 & -0.05 & -0.03 & -0.01 & -0.03 & $-0.05^{\mathrm{c}}$ & 0.02 & $0.05^{\mathrm{c}}$ & 0.04 & 1.00 & & \\
\hline $\begin{array}{l}\text { 13. Socially prescribed } \\
\text { perfectionism }^{2}\end{array}$ & $0.06^{\mathrm{c}}$ & $0.05^{\mathrm{c}}$ & -0.04 & $-0.11^{\mathrm{a}}$ & 0.01 & $0.08^{\mathrm{b}}$ & 0.05 & $0.05^{\mathrm{c}}$ & $-0.09^{\mathrm{a}}$ & $0.10^{\mathrm{a}}$ & -0.05 & $0.37^{\mathrm{a}}$ & 1.00 & \\
\hline 14. Parental burnout ${ }^{2}$ & $0.11^{\mathrm{a}}$ & $-0.07^{\mathrm{b}}$ & -0.00 & $-0.15^{\mathrm{a}}$ & $0.09^{\mathrm{a}}$ & $0.07^{\mathrm{b}}$ & -0.01 & $0.05^{\mathrm{c}}$ & 0.04 & $0.20^{\mathrm{a}}$ & $0.10^{\mathrm{a}}$ & $0.23^{\mathrm{a}}$ & $0.38^{\mathrm{a}}$ & 1.00 \\
\hline$M$ & 0.90 & 36.47 & 4.00 & 3.00 & 1.24 & 0.09 & 0.11 & 2.25 & 0.73 & 0.24 & 7.72 & 3.34 & 2.53 & 1.39 \\
\hline$S D$ & & 6.51 & & & 0.43 & & & 1.34 & 0.45 & & 3.73 & 0.75 & 0.93 & 1.19 \\
\hline
\end{tabular}

${ }^{1}$ Categorical variable

${ }^{2}$ Median for ordinary level variables (variables 3 and 4 ) and proportion of the sample for dichotomous variables (variables $1,6,7$, and 10 )

${ }^{3}$ Mean scores

${ }^{\mathrm{a}} p<0.001$

${ }^{\mathrm{b}} p<0.01$

${ }^{c} p<0.05$ 
subscales of parental burnout as indicators of latent parental burnout) and multidimensional perfectionism (self-oriented perfectionism and socially prescribed perfectionism as two distinct latent factors, including 4 and 3 observed items as indicators, respectively). The measurement model for parental burnout fitted the data well $\left(\chi^{2}(2)=16.91, p<0.001 ; C F I=\right.$ $1.00 ; R M S E A=0.07, S R M R=0.01)$.

The fit of the measurement model for multidimensional perfectionism was: $\chi^{2}(13)=232.36, p<0.001 ; C F I=0.94$; $R M S E A=0.09 ; S R M R=0.06$. Inspection of the modification indices suggested that by allowing (1) the residuals of items 1 and 4 of self-oriented perfectionism to correlate and (2) the residuals of items 1 and 2 of socially prescribed perfectionism to correlate would improve the fit of the model. After these content-wise specifications, the model fitted the data well $\left(\chi^{2}(11)=97.82, p<0.001 ; C F I=0.97\right.$; $R M S E A=0.07$; SRMR $=0.03)$.

Next, the unique role of different family background variables in parental burnout were tested. These variables included parent's gender, age, single parenthood, blended family, employment status, education level (four dichotomous variables describing different levels of education), number of children in the household, hours spent with children during the day, perceived financial situation of the family (four dichotomous variables describing different levels of financial resources), at least one young child at home (age $<6$ ), and special needs of the child. First, the correlations between these background variables and the latent burnout were examined. Then, a model including the night background variables (i.e., gender, age, single parenthood, employment status, number of children in the household, time spent with children, perceived poor financial situation of the family, perceived superior financial situation of the family, and special needs of the child) that were statistically significantly $(p<0.05)$ associated with the latent burnout as predictors of the latent parental burnout factor was tested. The fit of the model was good $\left(\chi^{2}\right.$ $(20)=92.15, p<0.001 ; C F I=0.99 ; R M S E A=0.05)$. The model is shown in Fig. 1.

The results (Fig. 1) showed that of all the tested background variables, only gender, parents' age, financial situation, employment status, and children's special needs had a unique impact on parental burnout: the younger the parent, the more parental burnout the parent reported. Moreover, parents reporting poor financial situation reported higher levels of parental burnout than others, whereas parents reporting superior financial situation reported lower levels of burnout than others. Mothers showed higher levels of parental burnout than fathers, and unemployed parents showed higher burnout than employed parents. Finally, parents with a child with a developmental delay or special needs reported higher parental burnout scores than others. The background variables explained a total of $8 \%$ of the variation in parental burnout $(p<0.001)$.
Next, latent factors indicating parents' self-oriented and socially prescribed perfectionism were included into the previous model as predictors of parental burnout. In this model, background variables were allowed to correlate with latent perfectionism variables. The fit of the model was $\operatorname{good}\left(\chi^{2}(79)=486.29, p<0.001 ; C F I=0.95 ; R M S E A=\right.$ 0.06). After adding multidimensional perfectionism, parent's gender was no longer statistically significantly associated with parental burnout, suggesting that its impact on parental burnout is mediated by perfectionism. Consequently, a final model including the indirect paths from gender to parental burnout through self-oriented and socially prescribed perfectionism was constructed $\left(\chi^{2}(80)=\right.$ 488.47, $p<0.001 ; C F I=0.95 ; R M S E A=0.05)$. The model is shown in Fig. 2.

The results (Fig. 2) showed, first, that of the perfectionism variables, socially prescribed perfectionism was uniquely associated with parental burnout: The higher the level of socially prescribed perfectionism the parents reported, the higher the level of parental burnout they demonstrated. Second, the impact of gender on parental burnout was found to be mediated by socially prescribed perfectionism (standardized indirect effect $=0.03, p=0.01$ ). Mothers reported higher levels of both self-oriented and socially prescribed perfectionism than fathers and, particularly due to their higher levels of socially prescribed perfectionism, they also showed higher levels of parental burnout. Of the other background variables, age, poor perceived financial situation, employment status, and children's special needs were still uniquely associated with parental burnout, although to a lesser extent than socially prescribed perfectionism. Overall, the included factors explained a total of $26 \%$ of the variation in parental burnout, with the strongest predictor being socially prescribed perfectionism.

As final step of analyses, the interaction term Socially prescribed perfectionism X Self-oriented perfectionism was tested by estimating the effect of this interaction term on latent parental burnout. The results showed statistically significant interaction effect (standardized estimate $=0.13$, $p<0.001$, which increased the explained variation of parental burnout from $0.26 \%$ to $0.29 \%$. The found interaction effect is visualized in Fig. 3.

The results (Fig. 3) demonstrated that socially prescribed perfectionism was associated with increased level of parental burnout, the association being particularly strong when parents simultaneously reported high levels of selforiented perfectionism.

\section{Discussion}

The purpose of the present study was to identify the key family background variables explaining parental burnout 
Fig. 1 Background variables as predictors of parental burnout: The results of the structural equation model (standardized estimates; only statistically significant predictors included into the model). Note. $* * * p<$ $0.001, * * p<0.01, * p<0.05$

Fig. 2 Background variables and multidimensional perfectionism as predictors of parental burnout: The results of the structural equation model (standardized estimates). $* * * p<$ $0.001, * * p<0.01, * p<0.05$
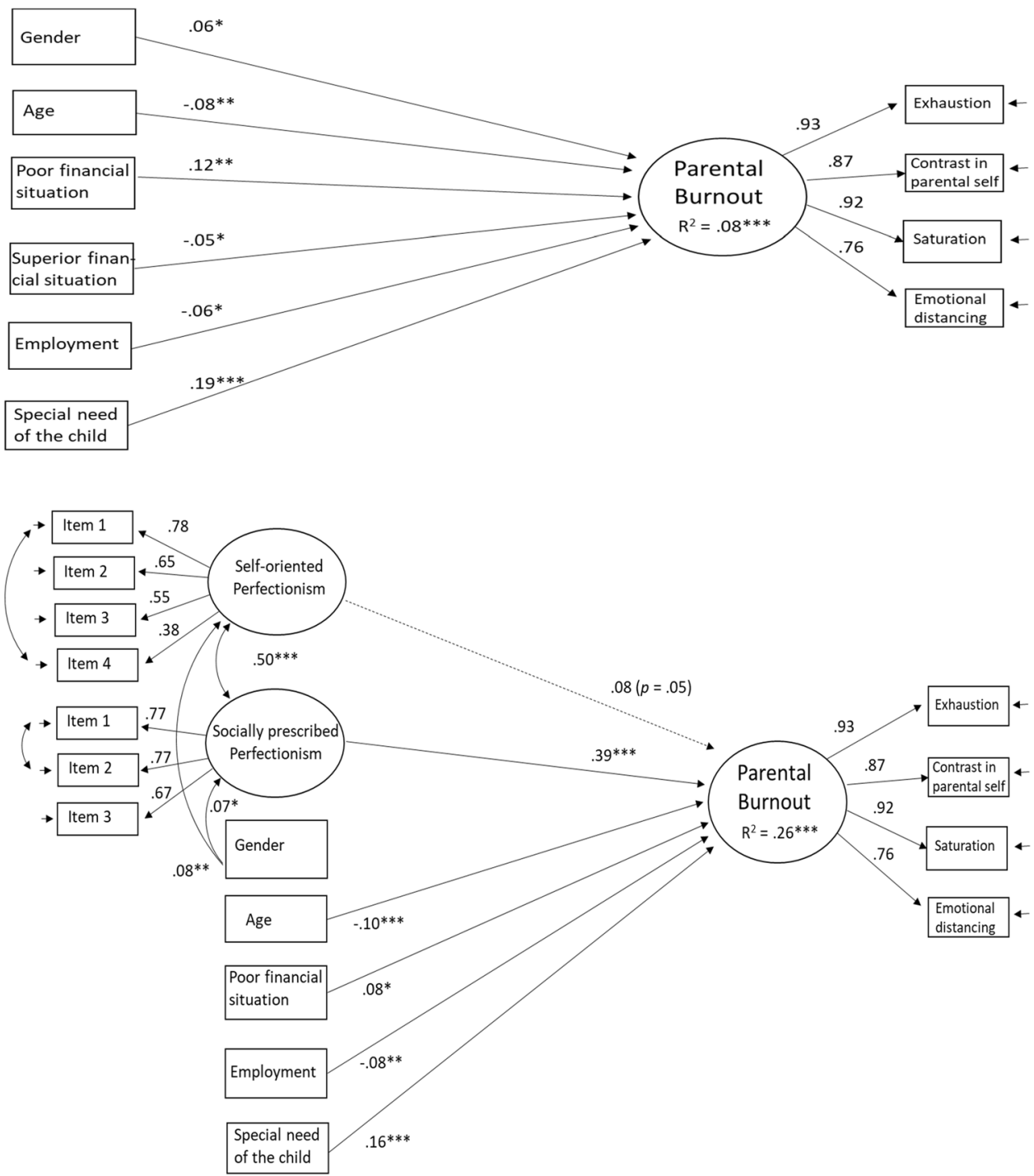

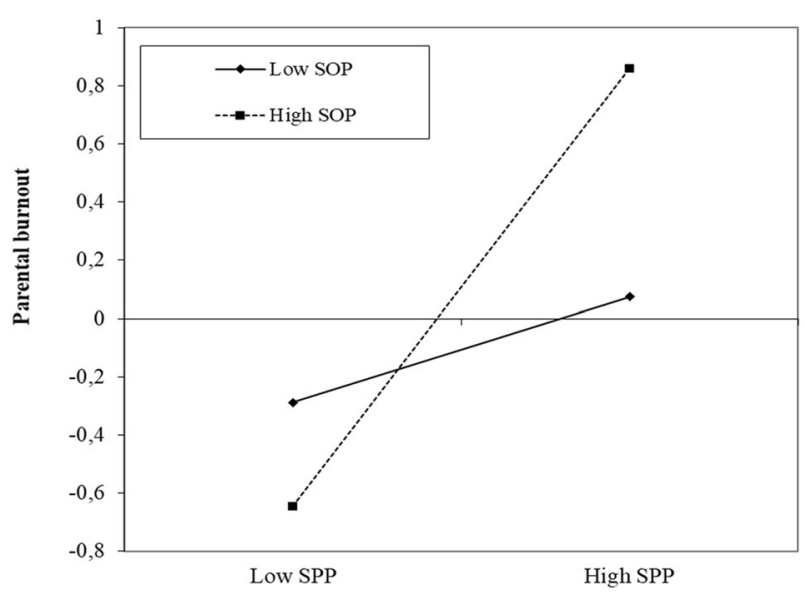

Fig. 3 The role of socially-prescribed perfectionism (SPP) in parental burnout among parents reporting low $(-1 S D$, low) and high $(+1 S D$, high) level of self-oriented perfectionism (SOP) among Finnish parents and to examine how self-oriented and socially prescribed perfectionism relate to parental burnout over and above the impacts of these background variables. The present study was the first study on parental burnout that was carried out among Finnish parents. The results showed that socially prescribed perfectionism, in particular, was a risk factor for parental burnout: the higher the level of socially prescribed perfectionism the parents reported, the higher the level of their parental burnout.

The first aim was to identify the key family background variables that explain parental burnout among Finnish parents. In Finland, people are highly educated (44\% of the population), females being more educated than males (Official Statistics of Finland 2018a). In the end of 2017, there were 1,472,000 families in Finland, which account for $74 \%$ of the Finnish population (Official Statistics of Finland 2018b). From families with children, the most common type is a nuclear family ( $81 \%$ of families), followed by single parent 
families (10\%) and blended families (9\%). Finnish families have 1.85 children, on average, and consist mostly of families with one child (43\%), followed by families with two children (39\%) and families with three children (13\%). Only 5\% of the families have more than five children (Official Statistics of Finland 2018b). After birth parents have a paid parental leave until the child is 10 months. Although Finnish parental leave policies are driven to improve gender-equality in parenting (Salmi and Lammi-Taskula 2014) and parental leave can be used by either of the parents, majority of the leaves is used by mothers.

The results showed that when family- and child-related background variables were all put into the same model as the explanatory variables, six of the variables remained significant: parent's gender, age of the parent, employment, perceived poor financial situation of the family, perceived superior financial situation of the family, and having a child with special needs. More specifically, mothers were more likely to be burned out than fathers, younger parents were more likely to be burned out than older ones, those who were unemployed were at a higher risk of burnout than those who were employed, those with poorer financial situations were more at risk than those with better financial situations, and those with superior financial situation were less at risk at burning out than those with worse financial situation. Finally, having a child with special needs was a risk factor for burnout. Some of these results are in line with previous findings. For example, previous research has shown that having a child with special needs is related to parental burnout, which can be explained by the increased investment and chronic stress of the parents (Lindström et al. 2010, 2011). Similarly, the results of the present study concerning the role of age in parental burnout are in line with earlier literature showing that younger age is also associated with parental burnout in Japan (Kawamoto et al. 2018) and France (Le Vigouroux and Scola 2018). It is possible that for younger parents there is a mismatch between the need for freedom and the commitments that family life requires, whereas older parents may be readier to settle down to raise children. In contradiction to the previous findings (Kawamoto et al. 2018; Le Vigouroux and Scola 2018; Mikolajczak et al. 2018b) being unemployed or having a low income were significant predictors of burning out as a parent. These effects may be specific to Finland, as it has been shown that economic poverty in Finnish families with children has been increasing during the past decade (Povlsen et al. 2018) and that poor families have significantly worse quality of life than those with better income (Karvonen and Salmi 2016). However, similar to the previous studies (Kawamoto et al. 2018; Le Vigouroux and Scola 2018; Mikolajczak et al. 2018) and in line with our expectations (Hypothesis 1), all family background variables together explained relatively little variance of parental burnout (8\%).
The second aim of the present study was to examine how multidimensional perfectionism is related to parental burnout over and above the impact of the background variables. The results showed that self-oriented perfectionism was only marginally related to parental burnout after taking the background variables and socially prescribed perfectionism into account. In previous studies conducted in occupational and sport settings, self-oriented perfectionism has been shown to be either negatively related to occupational (Childs and Stoeber 2010) and sport burnout (Appleton et al. 2009; Hill et al. 2008), or unrelated (Esposito et al. 2016; Gaudreau 2012). However, the relationship between parental burnout and perfectionism has been only been studied once previously (Kawamoto et al. 2018), and the results were similar to those of our study, showing that having high personal standards was unrelated to parental burnout. Overall, these results indicate that, in parenting settings, having high self-standards alone may not be a serious risk for burnout.

As expected (Hypothesis 2), socially prescribed perfectionism was positively related to parental burnout. Similar results have been found in sport (Appleton et al. 2009; Hill et al. 2008) and occupational settings (Childs and Stoeber 2010). Moreover, Kawamoto et al. (2018) showed that perfectionistic concerns were related to parental burnout, which also include worries about being evaluated negatively by others. In socially prescribed perfectionism, the excessively high standards are perceived to be imposed by others. Consequently, unlike in self-oriented perfectionism, individuals may feel that they have no control or agency. Furthermore, their self-worth may be dependent on the attainment of these perceived external standards. If they continue to fall short of these standards, they may be chronically exposed to anxiety and threat, which leads to burnout (Hill et al. 2008). Hill et al. (2008) suggested that, in a sport setting, socially prescribed perfectionists are "entrapped by a desire to validate a sense of self" (p. 633), and therefore they continue to participate long after they are physiologically drained. In parenting settings, parents cannot "quit" even when they are drained, but they can, however, become emotionally distanced from their children. Indeed, Roskam et al. (2018) proposed that such emotional distancing marks the final stage of parental burnout. Since research in the area of parental burnout is still in its infancy, future researchers are encouraged to look more deeply into these relationships by, for example, combining quantitative and qualitative methods, which can be particularly useful for unsearched and novel phenomena (see Sorkkila et al. 2018).

The results showed further that although the unique effect of self-oriented perfectionism on parental burnout was only marginal, in line with our expectations (Hypothesis 3) it demonstrated interaction effect with socially prescribed perfectionism: the effect of socially prescribed perfectionism on parental burnout strengthened in the presence of self-oriented 
perfectionism. The result is in line with the findings reported previously in clinical settings (Esposito et al. 2016) and suggests that it might be the combination of self-oriented perfectionism and socially prescribed perfectionism, in particular, that is detrimental to parents. It is possible that those individuals who think that being perfect is important (i.e., selforiented perfectionists) are more prone to internalize social pressure of ideal parenting than those who value selfperfection less (see Esposito et al. 2016) and, therefore, exhaust themselves as parents by constantly trying to do too well (see Lindström et al. 2011). Overall, inclusion of the interaction effect was found to increase the explained variance of parental burnout, and, as suggested by previous literature, seems thus to be important in understanding the nature and functionality of multidimensional perfectionism (Esposito et al. 2016; Gaudreau 2012).

Interestingly, when multidimensional perfectionism was added to the same model with the background variables that made unique contributions to parental burnout, gender was no longer a significant predictor of parental burnout. However, gender did have a small indirect effect on parental burnout through socially prescribed perfectionism. In other words, socially prescribed perfectionism mediated the relationship between gender and parental burnout: mothers reported more socially prescribed perfectionism than fathers, and consequently, they were also more burned out as parents. Noteworthy, mothers also reported higher levels of self-oriented perfectionism. There are several possible explanations for these findings. For example, there has been discussion about the "Myth of Motherhood," based on which unrealistically high expectations are placed on mothers by society, which shapes their experience as parents (fathers, in turn, are considered to be good parents simply by spending time with their children) (e.g., Douglas and Michaels 2004; Hagger 2011). Consequently, mothers may experience considerable guilt for not being perfect, as expected by themselves or by society (Hagger 2011). Douglas and Michaels (2004) suggested that "motherhood has become a psychological police state" (p. 6), meaning that in addition to societal judgment, mothers evaluate each other and themselves as mothers. Although these findings need to be replicated, it could be useful to promote public discussion on the expectations that are placed on mothers in today's society. Moreover, mothers could be taught skills such as self-compassion and acceptance, as recent studies have shown that unconditional self-acceptance may protect those exhibiting socially prescribed perfectionism from burnout (Hill et al. 2008). However, the reader must interpret this finding with caution, as the gender effect was only minor.

\section{Limitations}

This study had several limitations that should be taken into account when interpreting the results. First, although perfectionism has been largely viewed as a disposition that affects all domains (Hewitt and Flett 1991), recent evidence suggests that people may be perfectionists only in certain domains (Stoeber and Stoeber 2009). Consequently, it may be more accurate and precise to study parental perfectionism with a scale designed specifically for the parenting domain rather than one that is designed to measure overall perfectionism. Second, perfectionism was measured only as selforiented and socially prescribed perfectionism, which are most commonly used forms of perfectionism in the literature (see Stoeber 2014). However, the original scale of Hewitt and Flett (1991) also included a third aspect of perfectionism: other-oriented perfectionism. Other-oriented perfectionism entails expecting others to be perfect and being highly critical towards others failing to meet these expectations (Hewitt and Flett 1991). This aspect of perfectionism may be particularly relevant in the parenting context, as it can be detrimental to children. It would be interesting to examine how otheroriented perfectionism relates to parental burnout as well as to the burnout of children. Third, due to a long questionnaire battery, a shortened version of the perfectionism scale was used. Moreover, although the results of the present study provided support for factorial validity of the used scale and its translation, the scale has not yet been validated in a Finnish population. Fourth, this study was cross-sectional. Since burnout is a condition that evolves over time, it is crucial to investigate burnout across time using several measurement points (Sorkkila et al. 2018). Moreover, due to the lack of longitudinal follow up, it is not possible to draw any causal conclusions concerning, for example, the developmental dynamics of burnout and socially prescribed perfectionism. Thus, the results do not clarify whether the high standards expected from others make parents prone to burnout or whether burning out increases parents' tendency toward socially prescribed perfectionism. Fifth, many of the results were low of strength. In a large sample, even results that have little meaning in real-life may appear as significant. Consequently, it would be important to replicate these findings in other samples. Sixth, the number of fathers in the sample was low, and the participating parents were relatively highly educated; thus, the sample of fathers was not as representative as it could be. Finally, there were no clinical or other kinds of cut-off scores for parental burnout. Consequently, it is unknown what the results mean in real life. Future research should develop cut-off scores for parental burnout using, for example, descriptions of burned out parents, medical expertize, physiological measures (e.g., blood cortisol levels), and statements of significant others.

Notwithstanding the above-mentioned limitations, the present study offered novel information about parental burnout in Finland. The results suggest that there may be country-specific differences in the role played by sociodemographic background variables in parental burnout. In 
particular, in Finland, poor family financial resources and unemployment were significant predictors of parental burnout, which may be useful information to Finnish politicians and family decision-makers. However, similarly to other studies (Le Vigouroux and Scola 2018; Kawamoto et al. 2018; Mikolajczak et al. 2018b), the background variables explained relatively little of the variance in parental burnout $(8 \%)$. Socially prescribed perfectionism explained a larger proportion of the variance $(18 \%)$ and the effect of it strengthened even more if accompanied with self-oriented perfectionism. These results indicate that the combination of two types of perfectionism may be most detrimental for parents in terms of burning out. Parents, and particularly mothers, may therefore benefit from learning skills that aim at reducing self-blame stemming from high social expectations, such as self-acceptance and self-compassion.

Acknowledgements This study was funded by a grant from the Alli Paasikivi foundation (grant number: 21000041571), which was awarded to KA. Open access funding provided by University of Jyväskylä (JYU).

Author Contributions MS wrote the manuscript and received substantial input from KA. KA conducted the data analyses. The authors collected the data and designed the manuscript together.

\section{Compliance with Ethical Standards}

Conflict of Interest The authors declare that they have no conflict of interest.

Research Involving Human Participants and/or Animals Ethical permission for the study was obtained from the ethics commission of the University of Jyväskylä.

Informed Consent All participants filled in an informed consent prior to participation to the study.

Publisher's note Springer Nature remains neutral with regard to jurisdictional claims in published maps and institutional affiliations.

Open Access This article is distributed under the terms of the Creative Commons Attribution 4.0 International License (http://crea tivecommons.org/licenses/by/4.0/), which permits unrestricted use, distribution, and reproduction in any medium, provided you give appropriate credit to the original author(s) and the source, provide a link to the Creative Commons license, and indicate if changes were made.

\section{References}

Appleton, P. R., Hall, H. K., \& Hill, A. (2009). Relations between multidimensional perfectionism and burnout in junior-elite male athletes. Psychology of Sport and Exercise, 10, 457-465.

Aunola, K., Sorkkila, M., \& Tolvanen, A. (2019). Validity of the Finnish Version of the Parental Burnout Assessment (PBA). Manuscript submitted for publication.
Bakker, A. B., \& Demerouti, E. (2007). The job demands-resources model: state of the art. Journal of Management Psychology, 22, 309-326.

Baxter, L. A., Braithwaite, D. O., Bryant, L., \& Wagner, A. (2004). Stepchildren's perceptions of the contradictions in communication with stepparents. Journal of Social and Personal Relationships, 21, 447-467.

Bianchi, R., Truchot, D., Laurent, E., Brisson, R., \& Schonfeld, I. S. (2014). Is burnout solely job-related? A critical comment. Scandinavian Journal of Psychology, 55, 357-361.

Blanchard, L. T., Gurka, M. J., \& Blackman, J. A. (2006). Emotional, developmental, and behavioral health of American children and their families: a report from the 2003 national survey of children's health. Pediatrics, 117, 1202-1212.

Childs, J. H., \& Stoeber, J. (2010). Self-oriented, other-oriented, and socially prescribed perfectionism in employees: relationships with burnout and engagement. Journal of Workplace Behavioral Health, 25, 269-281.

Crnic, K., \& Low, C. (2002). Everyday stresses and parenting. In M. H. Bornestein (Ed.), Handbook of Parenting (pp. 243-268). Mahwah: Lawrence Erlbaum Associates.

Deater-Deckard, K. (2008). Parenting Stress. New Haven: Yale University Press.

Demerouti, E., Bakker, A. B., Nachreiner, F., \& Shaufeli, W. B. (2001). The job demands-resources model of burnout. Journal of Applied Psychology, 86, 499-512.

Douglas, D., \& Michaels, M. (2004). The mommy myth: the idealization of motherhood and how it has undermined all women. New York, NY: Free Press.

Esposito, R.M., Stoeber, J., Damian, L.E., Alessandri, G., \& Lombardo, C. (2016). Eating disorder symptoms and the $2 \times 2$ model of perfectionism: Mixed perfectionism is the most maladaptive combination. Eating and Weight Disorders, 24(4), 749-755.

Flett, G. L., Hewitt, P. L., Blankstein, K. R., \& O’Brien, S. (1991). Perfectionism and learned resourcefulness in depression and selfesteem. Personality and Individual Differences, 12, 61-68.

Flett, G. L., \& Hewitt, P. L. (2002). Perfectionism and maladjustment: an overview of theoretical, definitional, and treatment issues. In G. L. Flett \& P. L. Hewitt (eds), Perfectionism: Theory, Research and Treatment (pp. 5-31). Washington, DC: American Psychological Association.

Frost, R. O., Marten, P., Lahart, C., \& Rosenblate, R. (1990). The dimensions of perfectionism. Cognitive Therapy and Research, 14, 449-468.

Gaudreau, P. (2012). A methodological note on the interactive and main effects of dualistic personality dimensions: an example using the $2 \times 2$ model of perfectionism. Personality and Individual Differences, 52, 26-31.

Hagger, T. (2011). Making sense of an untold story: a personal deconstruction of the myth of motherhood. Qualitative Inquiry, $17,35-44$.

Hagqvist, E., Gådin, K. G., \& Nordenmark, M. (2017). Work-family conflict and wellbeing across Europe: the role of gender context. Social Indicators Research, 132(2), 785-797.

Hewitt, P. L., \& Flett, G. L. (1991). Perfectionism in the self and social contexts: conceptualization, assessment, and association with psychopathology. Journal of Personality and Social Psychology, 60, 456-470.

Hill, A. P., \& Curran, T. (2016). Multidimensional perfectionism and burnout: a meta-analysis. Personality and Social Psychology Review, 20, 269-288.

Hill, A. P., Hall, H. K., Appleton, P. R., \& Kozub, S. A. (2008). Perfectionism and burnout in junior elite soccer players: the mediating influence of unconditional self-acceptance. Psychology of Sport and Exercise, 9, 630-644. 
Karvonen, S. \& Salmi, M. (2016). Lapsiköyhyys Suomessa 2010luvulla. Helsinki: National Institute for Health and Welfare (Terveyden ja Hyvinvoinnin Laitos; THL). Article 30/2016.

Kawamoto, T., Furutani, K., \& Alimardani, M. (2018). Preliminary validation of Japanese version of the parental burnout inventory and its relationship with perfectionism. Frontiers of Psychology, 9, 970 .

Lammi-Taskula, J. (2017). Fathers on leave alone in Finland: negotiations and lived experiences. Comparative Perspectives on Work-Life Balance and Gender Equality, 6, 89-106.

Le Vigouroux, S., \& Scola, C. (2018). Differences in parental burnout: influence of demographic factors and personality of parents and children. Frontiers of Psychology, 9, 887.

Le Vigouroux, S., Scola, C., Raes, M.-E., Mikolajczak, M., \& Roskam, I. (2018). The big five personality traits and parental burnout: protective and risk factors. Personality and Individual Differences, 119, 216-219.

Lindhal-Norberg, A. (2007). Burnout in mothers and fathers of children surviving brain tumour. Journal of Clinical Psychology in Medical Settings, 14, 130-137.

Lindström, C., Åman, J., \& Lindhal-Norberg, A. (2010). Increased prevalence of burnout symptoms in parents of chronically ill children. Acta Paediatrica, 99, 427-432.

Lindström, C., Åman, J., \& Lindhal-Norberg, A. (2011). Parental burnout in relation to sociodemographic, psychosocial and personality factors as well as disease duration and glycemic control in children with Type 1 diabetes mellitus. Acta Paediatrica, 100, 1011-1017.

Lundberg, U., Måndberg., B., \& Frankenhaeuser, M. (1994). The total workload of male and female white collar workers as related to age, occupational level, and number of children. Scandinavian Journal of Psychology, 32, 151-170.

Maslach, C., \& Jackson, S. E. (1986). Maslach Burnout Inventory Manual. CA: Consulting Psychologists Press.

Maslach, C., Jackson, S. E., \& Leiter, M. P. (2001). Job burnout. Annual Review of Psychology, 52, 397-422.

Mikolajczak, M., Brianda, M. E., Avalosse, H., \& Roskam, I. (2018a). Consequences of parental burnout: Its specific effect on child neglect and violence. Child Abuse and Neglect, 80, 134-145.

Mikolajczak, M., Raes, M.-E., Avalosse, H., \& Roskam, I. (2018b). Exhausted parents: sociodemographic, child-related, parent-related, parenting and family-functioning correlates of parental burnout. Journal of Child and Family Studies, 27(2), 602-614.

Mikolajczak, M., \& Roskam, I. (2018). A theoretical and clinical framework for parental burnout: the balance between risks and resources $\left(\mathrm{BR}^{2}\right)$. Frontiers of Psychology, 9, 886.
Mills, J. S., \& Blankstein, K. R. (2000). Perfectionism, intrinsic vs. extrinsic motivation and motivated strategies for learning: a multidimensional analysis of university students. Personality and Individual Differences, 29, 1191-1204.

Molnar, D. S., Reker, D. L., Culp, N. A., Sadava, S. W., \& DeCourville, N. H. (2006). A mediated model of perfectionism, affect, and physical health. Journal of Research in Personality, 40, 482-500.

Muthén, L. K., \& Muthén, B. O. (2017). Mplus Users' Guide. 8th edn Los Angeles: Muthén and Muthén.

Official Statistics of Finland. (2018a). Educational structure of population. Helsinki: Statistics Finland. http://www.stat.fi/til/ vkour/2017/vkour_2017_2018-11-02_tie_001_en.html.

Official Statistics of Finland. (2018b). Families. Helsinki: Statistics Finland. http://www.stat.fi/til/perh/2017/perh_2017_2018-05-25_ tie 001 en.html.

Povlsen, L., Regber, S., Fosse, E., Karlsson, L. E., \& Gunnarsdottir, H. (2018). Economic poverty among children and adolescents in the Nordic countries. Scandinavian Journal of Public Health, 46, 30-37.

Roskam, I., Brianda, M.-E., \& Mikolajczak, M. (2018). A step forward in the conceptualization and measurement of parental burnout: the parental burnout Assessment (PBA). Frontiers of Psychology, 9, 1-12.

Roskam, I., Raes, M.-E., \& Mikolajczak, M. (2017). Exhausted parents: development and preliminary validation of the parental burnout inventory. Frontiers of Psychology, 8, 163.

Salmi, M., \& Lammi-Taskula, J. (2014). Policy goals and obstacles for fathers' parental leave in Finland. In Gu Eydal \& Tine Rostgaard, (Eds) Fatherhood in the Nordic Welfare states: comparing care policies and practice. Bristol: Policy Press.

Smith, M. M., Saklofske, D. H., Stoeber, J., \& Sherry, S. B. (2016). The big three perfectionism scale: a new measure of perfectionism. Journal of Psychoeducational Assessment, 34, 670-687.

Sorkkila, M., Ryba, T.V., Selänne, H., Aunola, K. (2018). Development of school and sport burnout in adolescent student-athletes: A longitudinal mixed methods study. Journal of Research on Adolescence. Advance of Print.

Stoeber, J. (2014). How other-oriented perfectionism differs from selforiented and socially prescribed perfectionism. Journal of Psychopathology and Behavioral Assessment, 36, 329-338.

Stoeber, J., \& Stoeber, F. S. (2009). Domains of perfectionism: prevalence and relationships with perfectionism, gender, age, and satisfaction with life. Personality and Individual Differences, 46, $530-535$. 